\title{
TERAPIA COMPORTAMENTAL COM FAMÍLIAS DE CRIANÇAS AGRESSIVAS: POR QUE, COMO E QUANDO'
}

\author{
Edwiges Ferreira de Mattos Silvares ${ }^{2}$
}

\begin{abstract}
RESUMO: Partindo de três perguntas-chave - por que, como e quando promover uma terapia com famílias de crianças agressivas - este trabalho objetiva demonstrar a relevância de uma intervenção envolvendo pais e crianças com problemas de conduta. É baseado na demanda crescente de atendimento infantil decorrente de dificuldades de natureza interpessoal, bem como na revisão da literatura, que aponta os estressores ambientais sobre a família e a ausência de habilidades cognitivas no repertório da criança como elementos a serem considerados na elaboração de estratégias clínicas. Estas devem ser aplicadas precocemente, a fim de se obter resultados mais eficazes. São descritas as atividades desenvolvidas pelo Programạ de Pós-graduação em Psicologia Clínica da Universidade de São Paulo - USP, envolvendo: 1) medidas de percepção dos pais e pares; 2) sessões terapêuticas com os pais e com as crianças; 3 ) análise da interação familiar, gravada em fitacassete pelos próprios pais. Também são apresentadas as atividades do grupo recreativo infantil desenvolvido na clínica-escola do Instituto de Psicologia da USP durante o processo terapêutico com a família.
\end{abstract}

Palavras-chave: agressividade infantil, modelagem de práticas parentais, intervenção preventiva

\section{BEHAVIORAL THERAPY APPLIED TO FAMILIES OF AGRESSIVE CHILDREN: WHY, HOW AND WHEN}

\begin{abstract}
Based upon three key questions - why, how and when to promote behavioral therapy with aggressive children's families, this study aims to demonstrate the relevance of a matched therapy apllied to parents and their child in cases of conduct disorders. It's based on the greater enrollement of children at Psychological Centers as a result of a poor relationship with peers, as well as on the review of the literature, which indicates the environmental stressors on parental caregiving and the lack of children's social cognition as cues for an intervention. The clinical strategies should be applied during early years, for more effective results in children's social adjustment. The activities that have been developped at the Psychology PostGraduation Program - São Paulo University are described, involving 1) perception of parents and peer group; 2) terapeutic sessions with parents and children; 3) analysis of family interactions, audio-recorded by the parents themselves. Activities developped by a children playing group in Psychology Institute during family intervention are also presented.
\end{abstract}

Key words: child disorder conducts, parent training, preventive intervention

É propósito deste trabalho comentar três pontos relativos ao processo de terapia comportamental com famílias de crianças agressivas, pontos estes que estão representados pelas questões que compõem o título do trabalho e dizem respeito às pesquisas que têm sido feitas sob orientação da autora do presente texto, desenvolvidas em grande parte no Laboratório de Terapia Comportamental

\footnotetext{
' Artigo recebido para publicação em 06/00; aceito em 04/01

2 Endereço para correspondência: Edwiges Ferreira de Mattos Silvares, Programa de Pós-Graduação em Psicologia Clínica, USP, Av Prof. Lúcio Martins Rodrigues, Trav. 5, Bloco 17, Cidade Universitária, São Paulo, SP, Cep 05508-900.e-mail efdmsilv@usp.br
}

da clínica-escola do Instituto de Psicologia da Universidade de São Paulo - IPUSP e vinculadas ao Programa de Pós-Graduação em Psicologia Clínica da USP.

Assim, ao término dessa leitura deverá ficar claro que o grupo de pesquisa coordenado pela autora, ao processar suas investigações científicas, tem se voltado para três perguntas principais, a saber:

1) Por que promover terapia comportamental com familias de crianças agressivas?

2) Como processar essa terapia de forma efetiva?

Paidéia, FFCLRP-USP, Rib. Preto, ago/dez/2000. 
3) Quando dar início a esse processo de intervenção clínica?

É esperado também que, no contato com as respostas, o leitor seja informado de forma breve acerca da evolução sofrida pela intervenção comportamental com famílias de crianças que têm dificuldades de interação por seus problemas comportamentais do tipo externalizante (Achenbach, 1991), ou seja, crianças agressivas.

Cumpre ainda lembrar que as respostas às questões antes colocadas serão discutidas de forma concisa e na ordem em que foram apresentadas, como deve também ficar explícito nessa seção, de modo a não estabelecer expectativas irreais para o leitor desavisado, que não há respostas acabadas para as questões propostas, visto que as considerações apresentadas constituem resultado de uma série de investigações científicas - brasileiras e estrangeiras algumas das quais ainda em desenvolvimento no Programa de Pós-Graduação em Psicologia Clínica da Universidade de S.Paulo, voltado para a formação de psicólogos clínicos-pesquisadores.

\section{Por que promover terapia comportamental com famílias de crianças agressivas?}

De acordo com McFadyen-Ketchum e Dodge (1998), a Psicologia tem deixado a responsabilidade de resolver os problemas de relacionamento interpessoal (como os enfrentados por crianças com queixa de agressividade, ou seja, com dificuldades de interação por seus problemas comportamentais do tipo externalizante) a escritores, educadores e vendedores ambulantes, da mesma forma que o sistema educacional também não parece preocupado com essa questão, como se estes problemas fossem irrelevantes ou passageiros.

Há, entretanto evidências de que o domínio de relações interpessoais positivas na infância, especialmente entre pares da mesma idade, é pré-requisito necessário para a saúde mental e o sucesso educacional futuros do indivíduo, como também é reconhecido que as relações infantis negativas com pares, pais e outros adultos estão associadas a resultados negativos na vida adulta, inclusive ao uso de substâncias ilegais e à violência interpessoal (McFadyen-Ketchum \& Dodge, 1998). E mais: arti- gos de revisão, como o de Parker e Asher (1987), claramente demonstram a força das relações entre rejeição social em idade tenra e os problemas sociais na vida posterior.

O reconhecimento da área de relacionamento interpessoal infantil como importante objeto de estudo é de interesse ainda maior se for levado em consideração também o que os estudos de caracterização da clientela infantil de clínicas-escola de Psicologia brasileiras (alguns desenvolvidos no Programa de Pós-Graduação, antes referido) têm mostrado, isto é, que a agressividade entre crianças encontra-se entre as três mais freqüentes queixas comportamentais apresentadas pela clientela infantil (Barbosa \& Silvares, 1994; Lopez, 1983; Silvares, 1991, 1996), sinalizando que a demanda por tal tipo de atendimento é uma imperiosa necessidade em nossos meios.

Do ponto de vista teórico e prático, por que orientar famílias de crianças agressivas é uma questão que exige atenção imediata, tanto pela falta de estudos empíricos brasileiros voltados para o problema, quanto pela demanda crescente de atendimento psicológico por parte da clientela infantil com dificuldades de natureza interpessoal. Felizmente, com o advento de intervenções comportamentais em programas delineados para melhorar as relações entre pares infantis e a eficácia no uso de certo tipo de medicação aconselhado para crianças hiperativas, capaz de reduzir a taxa dos comportamentos que interferem em suas relações sociais, a área começou a ter maior impacto sobre a saúde mental e o sistema educacional.

\section{Como processar terapia comportamental efetiva com familias de crianças agressivas?}

Fazer uma retrospectiva histórica da literatura da área é a maneira mais simples de encontrar a resposta empírica à questão de como orientar famílias de crianças agressivas, segundo objeto de nossa atenção neste trabalho.

Iniciando esta retrospectiva pelos diferentes estudos de Patterson, apresentados ao longo de mais de trinta anos de investigação sobre distúrbios de conduta em crianças americanas, chega-se ao trabalho elaborado por Patterson, Baryshe e Ramsey (1989). Estes autores, a partir dos seus diferentes 
estudos, construíram um modelo sobre o curso de desenvolvimento do comportamento anti-social. Para os autores citados, no período da infância inicial, as práticas parentais pobres em termos de disciplina $\mathrm{e}$ de monitoramento da criança propiciam as condições iniciais aos problemas de conduta. Estes problemas, por sua vez, levam a criança que freqüenta escola, na média infância, à rejeição pelo grupo de pares não desviante e a falhas acadêmicas. Estes dois aspectos interrelacionados acarretam o compromisso da criança que apresenta problemas de conduta com o grupo de pares desviante, o que subseqüentemente a encaminha para a delinquiência, no periodo da adolescência.

Esse modelo desenvolvimentista do comportamento anti-social leva quase naturalmente ao trabalho psicológico com pais das crianças agressivas, na tentativa de modificar as suas práticas parentais inadequadas que resultam no comportamento indesejável e anti-social dos seus filhos, culminando por induzi-los à delinqüência, quando se tornam adolescentes. Assim, não é de se admirar que a literatura sobre o treinamento de pais em práticas de manejo familiar tenha tido um crescimento surpreendente ao longo do tempo, chegando ao ápice na década de oitenta (Forehand \& Kotchick, 1996). Curiosamente, o patamar de interesse por essa literatura de treinamento de pais tem decrescido nos últimos anos, como se o domínio do conhecimento estivesse completo, o que não é verdadeiro, especialmente no que concerne à questão da diversidade cultural, ou seja, à orientação de pais pertencentes ao grupo dos carentes sociais.

E nessa última linha de raciocínio que devem ser lembrados novamente os nomes de Patterson e cols, (1989) que acrescentam outros elementos de compreensão ao modelo antes citado, ampliando-o. É o modelo ampliado que dá bases para um trabalho psicológico mais efetivo com crianças agressivas oriundas de grupos minoritários, visto que considera necessariamente os vários tipos de estressores ambientais atuantes sobre a família, capazes de contribuir para que o comportamento anti-social tenha o curso antes descrito.

Quatro tipos de variáveis têm o papel de interferência na interação familiar saudável, prejudicando-a do ponto de vista psicológico, a saber: 1) características de comportamento dos avós (ex: com- portamento anti-social e manejo familiar pobre), 2) características dos comportamentos dos pais (ex: comportamento anti-social e suscetivel a estressores), 3) variáveis sócio-demográficas (ex: baixa escolaridade, pertencer a grupo étnico minoritário) e 4) estressores familiares (ex: desemprego, conflito marital e separação cơnjugal).

A enorme influência desses estressores sobre o padrão de interação estabelecido pelas famílias de crianças com distúrbio de conduta foi observada a partir dos trabalhos de orientação comportamental com seus pais, nos quais os resultados da intervenção eram reconhecidamente positivos a curto prazo, mas não mostravam generalização ao longo do tempo. Assim, famílias carentes do ponto de vista sócio-econômico ou sob ação de qualquer outro tipo de estressor já mencionado, após treinamento psicológico em manejo das práticas parentais para torná-las mais consistentes, mais positivas, mais funcionais, menos coercivas e menos severas na disciplina de seus filhos, passavam a agir inicialmente de acordo com as orientações recebidas, mas não eram capazes de manter os ganhos ao encerrarem o trabalho com os psicólogos. Aliado a estes resultados, outras evidências empíricas - tais como as apontadas por Whaler e Dumas (1989) sobre as mães insulares - mostravam que a ação dos estressores sobre os familiares alteravam as percepções parentais, desfavorecendo sua interação com as crianças de conduta anti-social.

Além disso, uma vez que nas crianças agressivas não eram identificadas habilidades cognitivas específicas - uma ausência determinante dos problemas de relações sociais - e visto que respostas particulares deficientes em determinadas tarefas eram facilmente identificadas, muitos clínicos estavam prontos a tentar treiná-las, de modo a ampliar o repertório de respostas mais competentes. Assim, intervenções clínicas infantis orientadas para o reforçamento de respostas adequadas foram caracterizadas pelo foco neste nível. Entretanto, para padrões complexos de interação interpessoal (ex. explorar as similaridades e diferenças com um par) o reforçamento puro e simples de um comportamento desejado parecia não ser efetivo pois o comportamento necessário não se encontrava ainda no repertório da criança. Vários teóricos reconheceram então que estes comportamentos complexos ocorriam 
também em função de processos cognitivos mais sutis, tais como a habilidade da criança em reconhecer dicas externas relativas a pensamentos do colega ou antecipar as consequiências de certos comportamentos inadequados seus. Estes processos cognitivos passaram então a constituir um outro nível para o qual a intervenção psicológica precisava ser dirigida. Nesse campo de processamento de informação foram de particular importância os estudos de Crick e Dodge (1994), os quais haviam mostrado que as crianças socialmente competentes, em relação às incompetentes, eram mais:

a) efetivas na percepção das dicas internas e externas dos seus pares;

b) acuradas na percepção das dicas internas e externas dos seus pares e

c) eficazes na construção de objetivos que contribuiam para favorecer o relacionamento social com seus pares.

Desta forma, novas habilidades cognitivas foram focalizadas no trabalho de orientação psicológica com crianças anti-sociais, fruto da expansão do modelo de processamento de informação dos autores Crick \& Dodge (1994). Em outras palavras, houve uma ênfase maior sobre os processos cognitivos inadequados das crianças agressivas de modo a levá-las a encontrar formas de alterá-los durante a sua interação com colegas. Nessa medida, se antes destas descobertas os trabalhos com a criança agressiva se voltavam para o desenvolvimento de suas habilidades motoras e sociais, depois delas o desenvolvimento das habilidades cognitivas tiveram também lugar especial.

Esta breve retrospectiva histórica, finalizada com os estudos de Crick e Dodge (1994) e iniciada nos de Patterson e cols, (1989), mostra o caminho que Silvares e Marinho (1998), em seu artigo de revisão, já haviam sugerido, isto é, que o trabalho psicológico com crianças agressivas segue cada vez mais na direção de um atendimento psicológico conjugado no qual a família e a criança rẹcebem orientação psicológica visando melhorias no relacionamento interpessoal infantil. Nesse trabalho conjugado, onde criança e pais recebem orientação psicológica, déficits em habilidades cognitivas, motoras e emocionais são trabalhados com a criança, ao mesmo tempo em que os déficits nas práticas parentais de manejo familiar são abordados com os pais. Essa é a forma de trabalho que uma das mais recentes pesquisas de orientação de pais em nosso programa tem assumido, com resultados promissores (Baraldi, 2000).

De modo a poder avaliar a eficácia do trabalho de intervenção, levado a cabo com os pais e crianças com dificuldades de interação, são tomadas, antes, durante e depois da intervenção psicológica, medidas diversificadas e sucessivas, tanto do comportamento da criança como dos seus pais, em terapia e em interação na sua própria casa. Esta última forma de interação é gravada em fita cassete pela própria família, durante uma semana, em períodos diários de trinta minutos, enquanto as das sessões terapêuticas são gravadas em videocassete no Laboratório de Terapia Comportamental da clínica-escola do IPUSP. Os dois tipos de fita são transcritos e analisados em termos de frequiência de categorias comportamentais positivas e negativas da criança $e$ de seus familiares, consideradas da seguinte forma:

a) Categorias comportamentais positivas participação da criança nas tarefas domésticas, cumprimento de deveres escolares e manutenção de relações sociais com os familiares; observa-se respostas de aceitação e respeito pela criança, bem como o reforçamento dos comportamentos ditos positivos.

b) Categorias comportamentais negativas isolamento da criança e agressividade dirigida aos familiares e amigos; no caso dos familiares, registra-se a freqüência de comentários de desaprovação da criança, espancamentos, exigências acima de sua capacidade infantil.

Antes, durante e após a intervenção, também são tomadas medidas de percepção dos pais sobre o comportamento de suas crianças (pela resposta deles ao Child BehaviorCheck List - CBCL Achenbach, 1991) bem como de rejeição da criança pelos seus colegas, através da aplicação de escala sociométrica (Hops \& Greenwood, 1988).

A intervenção propriamente dita tem início com a discussão dali por diante. Estas regras são retomadas sempre que se observa a inclusão de novos contratos entre a terapeuta e as crianças, discutindose as regras que nortearão os trabalhos do grupo de 
integrantes. A partir daí, as sessões passam a enfocar o cotidiano das crianças, reforçadas no sentido de apresentarem aquilo que consideram como qualidades e habilidades na interação com seus colegas e familiares. Da mesma forma, começam a discutir alternativas para comportamentos classificados como inadequados.

Com o intuito de treinar as crianças na emissão de respostas de maior competência social, orientam-se as discussões para o reconhecimento das contingências relacionadas às situações caracterizadas pela agressividade, tomando-se casos do seu dia-adia, como brigas ocorridas na semana. Deste modo, elas aprendem, paulatinamente, não apenas a identificar as causas de seu comportamento, mas também a verificar as possibilidades de ação dentro de um mesmo contexto, ampliando seu repertório.

Através de estórias infantis, as crianças discutem, ainda, temas como cooperação e obediência, sendo preparadas para o treino de controle de ira. A constante retomada dos comportamentos - adequados e inadequados - ocorridos durante a semana e o caráter grupal da intervenção viabiliza o aprendizado das habilidades cognitivas necessárias ao desenvolvimento da competência social, objetivo maior deste trabalho terapêutico.

Com relação aos pais, a intervenção voltase para a discussão sobre as relações estabelecidas com os filhos, os comportamentos apresentados pela criança e formas de reforçamento daquelas respostas consideradas como adequadas. Verifica-se, en- tão, todo um processo de aprendizagem acerca do papel das contingências no controle do comportamento, seguido da avaliação das maneiras mais eficazes de se fornecer uma instrução. Além disso, conduz-se uma reflexão sobre a importância da atenção diferencial e do oferecimento de modelos adequados para a criança.

Nestas sessões de orientação, discute-se também a resolução de problemas e a qualidade da interação mantida com os filhos, como forma de alterar a percepção dos pais em relação às crianças e construir um repertório para o estabelecimento de interações menos coercitivas e mais satisfatórias.

Ainda na perspectiva preventiva de comportamentos anti-sociais, uma outra atividade desenvolvida refere-se ao grupo recreativo, que realiza brincadeiras e jogos lúdicos com as crianças quando, semanalmente, após terem sido atendidas em grupo pela psicóloga, aguardam o término da sessão de orientação de suas mães. Este trabalho, conduzido por um estagiário de Psicologia, visa agilizar a triagem e o diagnóstico das crianças encaminhadas com queixa de agressividade, bem como facilitar o vínculo com a instituição.

São apresentadas a seguir, com detalhes, as atividades que vêm sendo desenvolvidas na clínicaescola do IPUSP, durante as quinze sessões de terapia comportamental com um grupo de cinco pais e outras quinze sessões com os filhos que, por suas dificuldades de interação, foram encaminhados para atendimento.

Tabela 1: Objetivos das sessões terapêuticas com as crianças.

\begin{tabular}{|l|l|}
\hline \multicolumn{1}{|c|}{ SESSÕES } & \multicolumn{1}{c|}{ OBJETIVO } \\
\hline $1^{\mathrm{a}}$. sessão & $\begin{array}{l}\text { Apresentação do espaço, da terapeuta e das crianças. } \\
\text { Discussão sobre a função da clínica, da terapia e do psicólogo. } \\
\text { Discussão das regras do grupo. }\end{array}$ \\
\hline $2^{\mathrm{a}}$. sessão & $\begin{array}{l}\text { Retomada das regras. } \\
\text { Levantamento dos problemas que trouxeram as crianças à clínica e sua ocorrência. }\end{array}$ \\
\hline $3^{\mathrm{a}}$. sessão & $\begin{array}{l}\text { Levantamento de coisas boas que as crianças fazem e discussão de alternativas para os } \\
\text { comportamentos inadequados. }\end{array}$ \\
\hline $4^{\text {a } \text { sessão }}$ & $\begin{array}{l}\text { Conversa sobre alternativas de comportamento em situações de briga. } \\
\text { Discussão sobre em que momentos eles brigam, o que está acontecendo quando come- } \\
\text { ça a briga. }\end{array}$ \\
\hline $5^{\text {a } . \text { sessão }}$ & $\begin{array}{l}\text { Retorno das férias. } \\
\text { Apresentação dos novos integrantes. } \\
\text { Discussão das brigas que ocorreram na semana, o que aconteceu antes, o que aconte- } \\
\text { ceu depois e o que poderia mudar. }\end{array}$ \\
\hline
\end{tabular}




\begin{tabular}{|l|l|}
\hline $6^{\text {a }}$. sessão & $\begin{array}{l}\text { Discussão do tema cooperação através de leitura do livro João e Maria. } \\
\text { Retomada dos comportamentos-queixa que ocorreram durante a semana e alternativas } \\
\text { para estes. }\end{array}$ \\
\hline $7^{\mathrm{a}}$. sessão & $\begin{array}{l}\text { Discussão das dificuldades em se comportar da maneira discutida na última sessão e } \\
\text { treino de controle da ira. }\end{array}$ \\
\hline $8^{\mathrm{a}}$. sessão & $\begin{array}{l}\text { Discussão do tema obediência a partir da leitura de Chapeuzinho Vermelho. } \\
\text { Treino de controle de ira. } \\
\text { Identificação das dicas de que vai perder o controle. }\end{array}$ \\
\hline $9^{\mathrm{a}}$. sessão & $\begin{array}{l}\text { Discussão do tema cooperação e bondade através da leitura da história Galinha ruiva. } \\
\text { Discussão dos comportamentos que ocorreram na semana - adequados e inadequados. }\end{array}$ \\
\hline $10^{\mathrm{a}}$. sessão & $\begin{array}{l}\text { Discussão de uma estória inventada pelas crianças. } \\
\text { Discussão dos comportamentos que ocorreram na semana. }\end{array}$ \\
\hline $11^{\mathrm{a}}$. sessão & $\begin{array}{l}\text { Discussão de uma estória que as crianças inventam com intervenção da terapeuta. } \\
\text { Discussão dos comportamentos que ocorreram durante a semana - objetivando alter- } \\
\text { nativas possíveis aos inadequados. }\end{array}$ \\
\hline $12^{\mathrm{a}}$. sessão & $\begin{array}{l}\text { Discussão do tema obediência através da leitura de O Lobo e os sete cabritinhos. } \\
\text { Discussão dos comportamentos que ocorreram na semana. }\end{array}$ \\
\hline $13^{\mathrm{a}}$. sessão & $\begin{array}{l}\text { Discussão dos sentimentos das crianças através do jogo Brincando com as expressães } \\
\text { (O que é cada sentimento que aparece, quando eles sentem, o que fazem quando sen- } \\
\text { tem, o que podem fazer). } \\
\text { Discussão dos comportamentos que ocorreram na semana. }\end{array}$ \\
\hline $14^{\mathrm{a}}$. sessão & $\begin{array}{l}\text { Discussão sobre por que boas maneiras são importantes. } \\
\text { Discussão dos comportamentos que ocorreram na semana. }\end{array}$ \\
\hline $15^{\mathrm{a}}$. sessão & $\begin{array}{l}\text { Finalização do grupo. } \\
\text { Discussão dos ganhos de cada um, retomada das atividades feitas, discussão sobre o } \\
\text { que foi aprendido com cada estória. }\end{array}$ \\
\hline
\end{tabular}

Tabela 2: Objetivos das sessões de orientação aos pais.

\begin{tabular}{|l|l|}
\hline SESSÕES & \multicolumn{1}{c|}{ OBJETIVO } \\
\hline $1^{\mathrm{a}}$. sessão & $\begin{array}{l}\text { Apresentação das mães, motivo que as trazem à clínica. } \\
\text { Discussão das regras do grupo. } \\
\text { Orientação sobre a necessidade de gravar as fitas cassete (noção de relação). }\end{array}$ \\
\hline $2^{\mathrm{a}}$. sessão & $\begin{array}{l}\text { Levantamento da freqüência dos comportamentos-queixa. } \\
\text { Discussão das relações que estão se estabelecendo entre pais e filhos. } \\
\text { Atribuição de tarefa aos pais, instruídos a registrar comportamentos positivos das crian- } \\
\text { ças. }\end{array}$ \\
\hline $3^{\mathrm{a}}$. sessão & $\begin{array}{l}\text { Discussão sobre os comportamentos adequados apresentados pelas crianças. } \\
\text { Discussão sobre a experiência de observação dos pontos positivos dos filhos. } \\
\text { Discussão sobre como fortalecer esses comportamentos. }\end{array}$ \\
\hline $4^{\mathrm{a}}$. sessão & $\begin{array}{l}\text { Discussão sobre maneiras adequadas de reforçar comportamentos. } \\
\text { Discussão sobre a importância de ignorar os comportamentos inadequados, enfocando os } \\
\text { seguintes aspectos: o que é ignorar, como fazê-lo, o que sentem sobre isso. }\end{array}$ \\
\hline $5^{\mathrm{a}}$. sessão & $\begin{array}{l}\text { Retorno das férias. } \\
\text { Apresentação dos novos integrantes. } \\
\text { Discussão sobre o que acontece quando o comportamento inadequado é ignorado de for- } \\
\text { ma apropriada e o adequado é reforçado. }\end{array}$ \\
\hline
\end{tabular}




\begin{tabular}{|c|c|}
\hline $6^{\text {a. }}$ sessão & $\begin{array}{l}\text { Levantamento de como as mães dão instrução a seus filhos. } \\
\text { Discussão de diferentes tipos de instrução. } \\
\text { Apresentação de exemplos de formas eficazes de dar instrução. }\end{array}$ \\
\hline $7^{\mathrm{a}}$. sessão & $\begin{array}{l}\text { Discussão sobre a forma correta de dar instrução: especificação do que deve ser feito, } \\
\text { garantia de que a criança esteja ouvindo, clareza, fornecimento de uma instrução por vez, } \\
\text { estabelecimento de uma consequência e cumprimento das contingências apresentadas. }\end{array}$ \\
\hline $8^{\mathrm{a}}$. sessão & $\begin{array}{l}\text { Discussão sobre os efeitos observados após o uso dessa forma de instrução. } \\
\text { Discussão sobre a importância de ser contingente, cumprindo o que foi dito. }\end{array}$ \\
\hline $9^{\mathrm{a}}$. sessão & $\begin{array}{l}\text { Discussão sobre como foi a infância dos pais, como foram seus pais e o que sentiam. } \\
\text { Discussão sobre a importância dos modelos. }\end{array}$ \\
\hline $10^{\mathrm{a}}$. sessão & $\begin{array}{l}\text { Discussão sobre Atenção Diferencial. } \\
\text { Nova discussão sobre a importância de ignorar os comportamentos inadequados e reforçar } \\
\text { os adequados. } \\
\text { Orientações sobre como ignorar comportamentos inadequados. }\end{array}$ \\
\hline $11^{\mathrm{a}} \cdot$ sessão & $\begin{array}{l}\text { Nova discussão sobre a importância de ser contingente e conseqüente. } \\
\text { Levantamento de ações prazerosas realizadas com os filhos. }\end{array}$ \\
\hline $12^{\mathrm{a}}$. sessão & $\begin{array}{l}\text { Discussão sobre a importância de fornecer modelos adequados para as crianças. } \\
\text { Retomada das orientações sobre reforçamento de comportamentos adequados. } \\
\text { Incentivo à manutenção de atividades prazerosas com as crianças. } \\
\end{array}$ \\
\hline $13^{\mathrm{a}}$. sessão & $\begin{array}{l}\text { Discussão sobre os progressos observados nas crianças, tanto na terapia - exemplificando } \\
\text { o que é feito no grupo - quanto no contexto familiar. } \\
\text { Discussão sobre resolução de problemas. }\end{array}$ \\
\hline $14^{\mathrm{a}} \cdot$ sessão & $\begin{array}{l}\text { Nova discussão sobre resolução de problemas. } \\
\text { Discussão sobre contrato de reforçamento e como estabelece-lo com a criança. }\end{array}$ \\
\hline $15^{\mathrm{a}}$. sessão & Nova discussão dos progressos das crianças e finalização do grupo. \\
\hline
\end{tabular}

São apresentadas, ainda, as quinze sessões do grupo recreativo, nas quais são desenvolvidos jogos lúdicos com essas crianças quando, semanalmente, após o atendimento em grupo pela psicóloga, brincam sob a coordenação de um estagiário de Psicologia enquanto aguardam suas mães, que estão sendo orientadas. Este tipo de atendimento tem sido utilizado também como uma nova forma de julgar os progressos alcançados pela família e criança em função da terapia conjugada a que estão se submetendo e tem se mostrado um importante instrumento de avaliação diagnóstica (Bueno \& Silvares, 2000; Guerrelhas, 2000).

Tabela 3: Atividades desenvolvidas no grupo recreativo.

\begin{tabular}{|l|l|}
\hline SESSÃO & \multicolumn{1}{|c|}{ ATIVIDADES } \\
\hline $1^{\mathrm{a}}$. sessão & $\begin{array}{l}\text { Confecção dos crachás } \\
\text { Discussão do que vieram fazer na clínica } \\
\text { Jogo de bola em roda, falando as idades, comidas que preferem, quantos irmãos têm etc } \\
\text { Brincadeira de Morto-vivo } \\
\text { Jogo de Tiro ao alvo }\end{array}$ \\
\hline $2^{\text {a } . \text { sessão }}$ & $\begin{array}{l}\text { Jogo de memória com os nomes das crianças } \\
\text { Desenho do que fizeram durante a semana } \\
\text { Campeonato de Pebolim }\end{array}$ \\
\hline $3^{\mathrm{a}}$. sessão & $\begin{array}{l}\text { Brincadeira de Dança das cadeiras } \\
\text { Brincando com a casinha de bonecas } \\
\text { Jogo Imagem e Ação Júnior }\end{array}$ \\
\hline $4^{\mathrm{a}}$. sessão & $\begin{array}{l}\text { Jogo de memória } \\
\text { Brincadeira de Siga o mestre } \\
\text { Brincadeira de Morto-vivo }\end{array}$ \\
\hline
\end{tabular}




\begin{tabular}{|l|l|}
\hline $5^{\text {a: }}$ sessão & $\begin{array}{l}\text { Desenho em cartolina feito por todo o grupo } \\
\text { Brincadeira da Batata quente } \\
\text { Jogo com bola: a criança que estiver com a bola na mão falará o nome de uma cor, de um } \\
\text { carro, comida que gosta, quantos anos tem etc, dependendo da regra especificada } \\
\text { Brincadeira de mímica } \\
\text { Brincadeira de Siga o mestre }\end{array}$ \\
\hline $6^{\mathrm{a}}$. sessão & $\begin{array}{l}\text { Modelando argila } \\
\text { Desenho individual em folha de papel sulfite }\end{array}$ \\
\hline $7^{\mathrm{a}}$. sessão & $\begin{array}{l}\text { Jogo de futebol misto } \\
\text { Brincadeira de pega-pega chamada Gatos e Ratos }\end{array}$ \\
\hline $8^{\mathrm{a}}$. sessão & $\begin{array}{l}\text { Jogo de baralho: Rouba-Monte } \\
\text { Jogo de cricket } \\
\text { Variações do jogo de cricket: quem joga a bola mais longe, dentre outras }\end{array}$ \\
\hline $9^{\mathrm{a}}$. sessão & Brincadeira da Batata quente \\
\hline $10^{\mathrm{a}}$.sessão & Confeccionando fantoches com copos plásticos, massinha, tinta, barbante e outros materiais \\
\hline $11^{\mathrm{a}}$. sessão & Gincana \\
\hline $12^{\mathrm{a}}$. Sessão & Jogo de Pebolim \\
\hline $13^{\mathrm{a}}$. sessão & $\begin{array}{l}\text { Brincadeira da Dança das cadeiras } \\
\text { Jogo Lince } \\
\text { Jogo de memória }\end{array}$ \\
\hline $14^{\mathrm{a}}$. sessão & Jogo Imagem e Ação \\
\hline $15^{\mathrm{a}}$. sessão & $\begin{array}{l}\text { Jogo de Pebolim } \\
\text { Jogo Lince } \\
\text { Jogo de memória }\end{array}$ \\
\hline
\end{tabular}

Dessa forma, a segunda questão desse trabalho sobre 0 como orientar as famílias de crianças com dificuldades de interação, parece ter sido respondida pela descrição de uma das mais recentes pesquisas de orientação de pais atualmente em desenvolvimento sob orientação da autora (Baraldi, 2000), cujos resultados até o momento têm se mostrado promissores. Entre os principais pontos deste trabalho, em fase de finalização, merecem destaque: 1) a alteração positiva das percepções dos pais acerca do comportamento de seus filhos, obtidas através dos CBCLs e verificadas após a intervenção; 2) a redução das interações negativas entre pais e filhos registradas em fita cassete no ambiente doméstico, seguida do aumento das interações positivas e 3) a redução dos comportamentos inadequados das crianças no grupo recreativo, observada à medida que a intervenção terapêutica se deseñvolve.

\section{Quando dar início a esse processo de orientação de familias}

De tudo que foi até aqui exposto decorre a resposta natural e direta à terceira questão colocada Paidéia, FFCLRP-USP, Rib. Preto, ago/dez/2000. no início deste, ou seja: o trabalho psicológico com familias de crianças agressivas deve se dar o mais cedo possivel, antes mesmo que a criança tenha ingressado na média infância.

O trabalho de orientação de pais. de crianças agressivas na clínica-escola do IPUSP, entretanto, tem abrangido crianças de 6-10 anos - a faixa etária modal dà instituiçắo (Silvares, 1991), quando elas já estão em plena atividade escolar, pois a grande maioria é encaminhada à instituição nessa idade.

Considerando, porém, as colocações feitas nas duas seções anteriores, que apontam a necessidade de intervenção com crianças de famílias de risco antes que os problemas de comportamento delas tenham se instalado, já foi desenvolvido por uma doutoranda da equipe da autora um trabalho preventivo com grupo de pais que reconheciam dificuldades de interação com seus filhos, antes mesmo que outros agentes sociais como os professores as tivessem apontado. De modo a impedir a evasão do atendimento, o programa preventivo foi até a comunidade, isto é, na sucursal da clínica-escola do IPUSP em um bairro periférico da cidade de São Paulo, onde 
tiveram lugar as sessões de terapia comportamental a pais e filhos. Os resultados desse trabalho foram bem satisfatórios do ponto de vista de alcançar com os pais um melhor manejo de práticas parentais e, conseqüentemente, a produção de alterações nos padrões de comportamento das crianças envolvidas no trabalho (Mello, 1998). O único ponto de insatisfação com esse programa foi o pequeno número de pessoas nele envolvidas, fazendo-se necessário desenvolver um esquema de trabalho preventivo que consiga alcançar essas crianças e seus pais de forma mais abrangente, o que se encontra atualmente em desenvolvimento (Mello, 2000).

\section{Referências Bibliográficas}

Achenbach, T.M. (1991). Integrative guide for the 1991 CBCL/ 4-18, YSR, and TRF Profiles. Burlington: University of Vermont.

Baraldi, D. (2000). Orientaçâo remediativa de pais de crianças agressivas numa clínica-escola (relatório n. $02 \mathrm{CNPq}$ ). São Paulo: Universidade de São Paulo.

Barbosa, J.I.C. \& Silvares, E.F.M. (1994). Caracterização preliminar das clínicas-escola de Psicologia de Fortaleza. Estudos de Psicologia, 28(1), 10-14.

Bueno, M.O. \& Silvares, E.F.M. (2000, abril). Grupo de espera recreativo: uma alternativa de atendimento, uma nova forma de avaliação diagnóstica. Poster apresentado no I International Symposium of Sciente Initiation. New Jersey.

Crick, N.R. \& Dodge, K.A. (1994). A review and reformulation of social information -processing mechanisms in children's social adjustment. Psychological Bulletin, 115 (1), 74-10.

Forehand, R. \& Kotchick, B.A. (1996). Cultural Diversity: A wake-up call for parent training. Behavior therapy, 27, 187-206.

Guerrelhas, F.F. (2000). Lista de espera $x$ grupos de espera recreativos: uma experiência numa clinica-escola de São Paulo. Dissertação de Mestrado, Universidade de São Paulo, São Paulo.
Hops, H. \& Greenwood, C.R. (1988). Social skills deficits. Em E. J. Mash \& L.G. Terdal (Eds.), Behavioral assessment of childhhod disorders (pp. 263-316). New York: Guilford Press.

Lopez, M.A. (1983). Características da clientela de clinicas - escola de psicologia em São Paulo. Arquivos Brasileiros de Psicologia, 35 (1), 78-92.

McFadyen-Ketchum, S.A. \& Dodge, K.A. (1998). Problems in Social Relationships. Em E.J. Masch \& R.A. Barkley (Orgs.), Treatment of Childhood Disorders(pp. 338-369). New York: Guilford Press.

Mello, M.H.S. (1998). Orientação preventiva de pais numa clínica-escola. Dissertação de Mestrado, Universidade de São Paulo, São Paulo.

Mello, M.H.S. (2000). Orientação preventiva de pais de crianças com dificuldades de interação numa clínica-escola. (relatório n. 02 CAPES), São Paulo, Universidade de São Paulo.

Parker, R.D. \& Asher, S.R. (1987). Peer acceptance and later personal adjustment: Are low accepted children at risk? Psychological Bulletin, 102, 357-389.

Patterson G.R., Baryshe, B.D. \& Ramsey (1989). A developmental perspective on antisocial behavior. American Psychologist, 44(2), 329335.

Silvares, E.F.M. (1991). O atendimento comunitário nas clínicas-escola de Psicologia [Resumo]. Em Anais do XXIII Congresso Interamericano de Psicologia (pp. 408-418), Costa Rica.

Silvares, E.F.M. (1996). É satisfatório o atendimento psicológico nas clínicas-escola brasileiras? Coletâneas da ANPEPP, 137-147.

Silvares, E.F.M \& Marinho, M.L. (1998). Ampliando la intervención psicológica a la familia en la terapia conductual infantil. Psicologia Conductual 6 (3), 617-627.

Whaler, R. G. \& Dumas, J. E. (1989). Attentional problems in dysfunctional mother-child interactions: An interbehavioral model. Psychological Bulletin, 105, 116-130.

Paidéia, FFCLRP-USP, Rib. Preto, ago/dez/2000. 